Ethics Approval This study was approved by the following Ethics Committees: COMITE DE PROTECTION DES PERSONNES, Sud-Méditerranée V (Gustave Roussy, IPC), Comité d'Ethique Institut Jules Bordet, COMITÉ DE ÉTICA DE INVESTIGACIÓN CLÍNICA CON MEDICAMENTOS del Hospital Universitari Vall d'Hebron, Ethikkommission an der TU Dresden, HRA London-Surrey Borders Research Ethics Committee.

Consent Written informed consent was obtained from the patient for publication of this abstract and any accompanying images. A copy of the written consent is available for review by the Editor of this journal.

http://dx.doi.org/10.1136/jitc-2020-SITC2020.0316

\section{A PHASE 1/1B STUDY OF SBT6050, A HER2-DIRECTED MONOCLONAL ANTIBODY CONJUGATED TO A TOLL- LIKE RECEPTOR 8 AGONIST, IN SUBJECTS WITH ADVANCED HER2-EXPRESSING SOLID TUMORS}

\begin{abstract}
${ }^{1}$ Leisha Emens*, ${ }^{2}$ Muralidhar Beeram, ${ }^{3}$ Erika Hamilton, ${ }^{4}$ Sarina Piha-Paul, ${ }^{5}$ Valerie Odegard, ${ }^{5}$ Sue Hamke, ${ }^{5}$ Naomi Hunder, ${ }^{6}$ Samuel Klempner. ' University of Pittsburgh Medical Center, Pittsburgh, PA, USA; ${ }^{2}$ The Start Center for Cancer Care, San Antonio, TX, USA; ${ }^{3}$ Sarah Cannon Research Institute, Nashville, TN, USA; ${ }^{4} M D$ Anderson Cancer Center, Houston, TX, USA; ${ }^{5}$ Silverback Therapeutics, Inc., Seattle, WA, USA; ${ }^{6}$ Massachusetts General Hospital, Boston, MA, USA
\end{abstract}

Background New strategies are needed to improve outcomes in human epidermal growth factor receptor 2 (HER2)expressing cancers. SBT6050 is a novel therapeutic comprising a specific small molecule toll-like receptor (TLR) 8 agonist conjugated to a HER2-directed monoclonal antibody. TLR8 is highly expressed in myeloid cells that are prevalent in human tumors, including dendritic cells (DCs) and macrophages, and modulates their pro-inflammatory activity. SBT6050 is designed to activate human myeloid cells only in the presence of moderate-to-high HER2 expression (immunohistochemistry [IHC] $2+$ or $3+$ ) and binds to the same epitope as pertuzumab. In preclinical studies, SBT6050 potently induces a broad spectrum of antitumor immune mechanisms, including proinflammatory cytokine and chemokine production, inflammasome activation, and indirect activation of $\mathrm{T}$ and natural killer (NK) cells. TLR8 agonism has emerged as a promising approach to overcome resistance to immune checkpoint inhibitors in tumors lacking Tcell infiltrates, as these cancers are often replete with myeloid cells. Using an SBT6050 mouse surrogate in vivo, curative single-agent efficacy was observed in multiple murine tumor models, including a model deficient in T, B, and NK cells. In preclinical toxicology studies in nonhuman primates, SBT6050 was well tolerated, supporting a first-inhuman starting dose that is predicted to be pharmacologically active, with a short escalation to projected clinically active doses. Preclinical studies also support combinations with checkpoint inhibitors and with trastuzumab to further enhance antitumor activity.

Methods SBT6050-101 is an ongoing phase $1 / 1 \mathrm{~b}$, first-inhuman, open-label, multicenter study. Eligible subjects are adults with histologically confirmed, HER2-expressing (IHC 2 + or $3+$ ), locally advanced (unresectable) and/or metastatic cancer. Subjects must have measurable disease per the Response Evaluation Criteria in Solid Tumors (RECIST) 1.1 and have previously received all therapies known to confer clinical benefit. SBT6050 is given subcutaneously every 2 weeks and treatment may continue for up to 2 years or until disease progression, unacceptable toxicity, or other reason for discontinuation. The trial objectives are to evaluate the safety and tolerability of SBT6050 and to identify the maximum tolerated dose and recommended phase 2 dose (RP2D). The study has 2 parts: Part 1, consisting of a dose escalation using a standard $3+3$ design, and Part 2, consisting of 5 parallel expansion cohorts based on tumor type and HER2 expression level and treated with SBT6050 at the RP2D. Pharmacokinetics, immunogenicity, and antitumor activity will be evaluated and pharmacodynamic markers of myeloid cell activation will be assessed in peripheral blood and on-treatment tumor biopsies.

Results N/A

Conclusions N/A

Trial Registration NCT04460456

Ethics Approval The study was approved by MD Anderson Cancer Center Institutional Review Board, approval number 2020-0326 MOD001.

http://dx.doi.org/10.1136/jitc-2020-SITC2020.0317

\section{OLAPARIB PLUS PEMBROLIZUMAB IN PATIENTS WITH PREVIOUSLY TREATED ADVANCED SOLID TUMORS WITH HOMOLOGOUS RECOMBINATION REPAIR MUTATION AND/OR HOMOLOGOUS RECOMBINATION REPAIR DEFICIENCY: KEYLYNK-007}

${ }^{1}$ Timothy A Yap*, ${ }^{2}$ Mallika Dhawan, ${ }^{3}$ Andrew E Hendifar, ${ }^{4}$ Michele Maio, ${ }^{5}$ Taofeek K Owonikoko, ${ }^{6}$ Miguel Quintela-Fandino, ${ }^{7}$ Ronnie Shapira-Frommer, ${ }^{8}$ Sanatan Saraf, ${ }^{8}$ Ping Qiu, ${ }^{8}$ Fan Jin, ${ }^{8}$ Alexander Gozman, ${ }^{9}$ Douglas A Levine. ${ }^{1}$ The University of Texas MD Anderson Cancer Center, Houston, TX, USA; ${ }^{2}$ Helen Diller Family Comprehensive Cancer Center, University of California San Francisco, San Francisco, CA, USA; ${ }^{3}$ Samuel Oschin Cancer Center, Cedars-Sinai Medical Center, Los Angeles, CA, USA; ${ }^{4}$ Center for ImmunoOncology, University Hospital of Siena, Siena, Italy; ${ }^{5}$ Winship Cancer Institute, Emory University, Atlanta, GA, USA; ${ }^{6}$ Centro Nacional de Investigaciones Oncológicas, Madrid, Spain; ${ }^{7}$ Chaim Sheba Medical Center, Tel HaShomer, Israel; ${ }^{8}$ Merck and Co., Inc., Kenilworth, NJ, USA; ${ }^{2}$ Laura and Isaac Perlmutter Cancer Center, New York, NY, USA

Background Treatment with the anti-PD-1 antibody pembrolizumab has improved clinical outcomes in multiple previously treated advanced solid tumors. The poly (ADP-ribose) polymerase (PARP) inhibitor olaparib has shown antitumor activity as monotherapy in patients with previously treated advanced ovarian, breast, pancreatic, and prostate cancers with BRCA1/BRCA2 mutations (BRCAm). Activity was also seen in patients with previously treated advanced solid tumors with other homologous recombination repair mutation (HRRm) and in those with ovarian cancer with homologous recombination repair deficiency (HRD) phenotype. PARP inhibitors have been found to increase interferon signaling and tumor infiltrating lymphocytes, enhancing tumor susceptibility to immune checkpoint blockade. Antitumor activity of PD-(L)1 plus PARP inhibition was found to be higher than expected with either agent alone in patients with recurrent ovarian cancer regardless of BRCAm or HRD status and in patients with BRCAm breast cancer. KEYLYNK007 (NCT04123366) evaluates the antitumor activity and safety of olaparib in combination with pembrolizumab in patients with previously treated advanced solid tumors with HRRm and/or HRD.

Methods This phase 2, nonrandomized, multicenter, open-label study will enroll approximately 300 patients aged $\geq 18$ years 